\title{
Comunicación y juego. Usos del lenguaje infantil en diferentes situaciones de juego
}

\author{
Pilar Nicolau
}

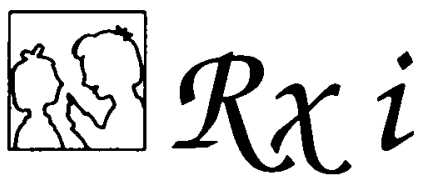

Las diferentes situaciones de juego en las que participan los niños en edad infantil, se convierten en instrumento capaz de provocar y modificar diferentes tipos de intercambios comunicativos. Si el juego es un formato de comunicación e interacción donde el conocimiento se comparte y reestructura, estos formatos (juegos) serán responsables en gran medida de la cantidad y diversidad de diálogos que en ellos se puedan crear. Este trabajo que se presenta aborda esta cuestión, aportando datos sobre observaciones en clase, a la vez que reflexiona sobre el potencial comunicativo de las situaciones de juego (o la organización del material didáctico) como elemento de análisis para la práctica educativa de la comunicación verbal infantil.

\section{IINTRODUCCION}

Es ya en hecho evidente que los niños de 3, 4 ó 5 años poseen un dominio de gran parte de la complejidad del sistema lingüístico que usan y ponen en funcionamiento en diferentes contextos y situaciones. Pero también es verdad que la escuela se convierte a partir de estas edades en uno de los contextos donde las posibilidades de interacción se amplían considerablemente dando lugar a un amplio abanico de posibilidades de comunicación (los niños se comunican en el patio, en la clase, con la maestra, con otros niños...) cuyo resultado son diferentes tipos de diálogos que pueden variar no sólo en longitud del intercambio verbal, sino también en lo que se refiere a los diferentes usos que hacen del lenguaje.

\section{PERSPECTIVA TEORICA}

Lenguaje, aprendizaje y comunicación

Los postulados teóricos sobre los que se basa este análisis son dos. En primer lugar, la tesis de Piaget sobre el principio de observación y actividad como base del aprendizaje. Piaget (1975) sostiene que el aprendizaje se inicia con la información 
que se obtiene a través de los sentidos. De esta forma, el niño aprende a conocer el mundo que le rodea a partir de su propia acción (observa, manipula, examina diferentes objetos y materiales), y es esta interacción con los objetos la que le proporciona la información necesaria para la adquisición del conocimiento.

En segundo lugar, si bien la experiencia concreta proporciona información del exterior, es solo una parte de la experiencia global del niño. Siguiendo a Vygotski (1979), la acción del niño sobre el objeto es fuente de información, pero sin olvidar que la mayor parte del tiempo que el niño dedica a explorar, manipular, observar, lo hace inmerso en intercambios sociales con otras personas, siendo la conversación, el intercambio más relevante de la interacción. Esta afirmación nos lleva a pensar que no es la actividad solitaria del niño la que le proporciona el conocimiento del mundo exterior, sino también, la relación que establece con las personas le sirve de ayuda en la elaboración de este conocimiento.

El lenguaje es una representación de la realidad, y es precisamente este caracter simbólico lo que le confiere su función comunicativa original. Las primeras palabras del niño son producto de intercambios comunicativos con otras personas, pero su significado va evolucionando en la medida que se usan en otros contextos. Las palabras ayudan al niño a formar conceptos $y$, a su vez, su significado evoluciona posibilitando nuevos usos del lenguaje.

Vygotski señala que el aprendizaje de muchos conceptos no puede desarrollarse a partir de la actividad directa, sino que es a través del lenguaje como se puede ayudar al niño en la comprensión de conceptos abstractos que no son fácilmente observables.

Si para Vygotski los intercambios comunicativos del niño representan el medio a través del cual se facilita el aprendizaje y desarrollo conceptual, Piaget y sus colaboradores señalan que el pensamiento surge a partir de las propias acciones. Sin ánimo de apostar por uno u otro planteamiento, ambos aportan elementos en la configuración del desarrollo cognitivo. En interacción con otras personas, la conversación confiere significado a las acciones del niño y, a su vez, éstas proporcionan la base esencial para que los significados se asocien al lenguaje (Tough, 1979).

\section{Comunicación y relaciones entre iguales}

El lenguaje permite comunicar e intercambiar significados de forma breve que no proporciona otro medio de comunicación, (es más, algunos significados sólo puden ser expresados a través del lenguaje), conceptos o ideas complejas pueden comunicarse con una sola palabra. Considerándolo bajo este concepto, el desarrollo del lenguaje estará relacionado con la creciente habilidad del niño para utilizar el sistema lingüistico como transmisor de significados (Tough, 1976). El lenguaje es el medio por el que èl pensamiento puede ser expresado, las ideas se transmiten de unas personas a otras.

En el período preverbal, el niño es capaz de transmitir significados a partir de gestos que progresivamente irá sustituyendo por palabras. Con el desarrollo del lenguaje, adquirirá un sistema de signos que le ampliará las posibilidades de comunicación. Las palabras y las reglas gramaticales serán èl instrumento que le ayudarán a expresar significados de diversa índole. Es fácil pensar que los progresos verbales se dan a partir de contextos comunicativos diversos en los que el niño aprende a actuar y a hablar de diferentes maneras. Primero serán las relaciones familiares las que le ayudarán en la construcción del desarrollo verbal y, posteriormente en la escuela, las nuevas situaciones le obligarán a aprender nuevas estrategias verbales para ir adaptándose a nuevas situaciones y contextos. El aprendizaje y desarrollo del lenguaje le 
permitirá construir y dominar el complejo sistema de significados. El conjunto de relaciones comunicativas se amplía considerablemente facilitando su proceso de socialización a la vez que va ampliando sus habilidades de comunicación.

En las escuela, los niños de 3 y 4 años pasan gran parte del tiempo jugando con los compañeros de su misma clase. Estos juegos pueden estar organizados por la maestra a partir de un aspecto concreto de la programación, o ser juegos que surjan de la propia espontaneidad del niño (eligiendo el material y los compañeros). En ambos casos, el juego se convierte en formato de comunicación e interacción donde el habla deviene instrumento de intercambio comunicativo que ayuda a reestructurar y definir la situación, aceptando acuerdos mutuos y posibilitando que el juego se vaya desarrollando.

Generalmente, en el transcurso del juego los niños hablan entre sí sobre diferentes aspectos, es decir, usan el lenguaje para expresar intenciones, propósitos, que van intercambiando entre sí dando lugar a conversaciones diversas según sea la información que se desea transmitir. Son muchas las situaciones en las que los niños utilizan el lenguaje en la escuela: cuando llegan a la clase, cuando discuten con sus compañeros, cuando piden permiso al profesor para hacer algo, cuando juegan con otros niños... Del conjunto de situaciones escolares, es esta última la que forma parte del objetivo de este análisis.

Tough (The Development of Meaning, 1977) presenta un resumen de una investigación sobre algunas diferencias en las expresiones infantiles en la utilización del lenguaje. Esta investigación aporta datos significativos sobre diferencias en el uso del lenguaje y experiencias lingüísticas familiares distintas. Los resultados reflejan claras diferencias en lo que respecta no sólo a la longitud media de las locuciones utilizadas, sino también diferencias significativas en relación a los propósitos con los que los niños utilizaban su lenguaje (desde usos del lenguaje para informar sobre lo que estaban haciendo, hasta usos para evocar experiencias pasadas, colaborar, anticipar...).

\section{OBJETIVO}

Es en este punto donde se situa el objetivo de esté análisis. Si, como plantea Tough, las experiencias lingüísticas favorecen determinados usos del lenguaje, es fácil pensar que en el colegio son muchas y variadas las situaciones en las que los niños hablan y se relacionan con los demás. De todas ellas, las situaciones de juego con los demás niños y las posibles diferencias en la utilización del lenguaje ha sido el objetivo de este estudio.

El planteamiento del análisis queda pues, resumido en dos preguntas:

- ¿ Los diferentes tipos de juego pueden considerarse como diferentes experiencias lingüísticas que dan lugar a diferencias en el uso del lenguaje?.

- Si es así, ¿cuáles son estas diferencias?.

\section{METODOLOGIA Y TRATAMIENTO DE DATOS}

\section{Sujetos}

La muestra estuvo formada por dos parejas de niños. La primera (Xisco e Isaac) con edades comprendidas entre los 3 años y 3 años y medio, la segunda (Imma y Laura) de 4 años y 4 años y medio. Ambas parejas de habla catalana. 


\section{Procedimiento}

Para dar respuesta a las dos preguntas del objetivo, se hicieron dos tipos de análisis. En primer lugar, se analizaron los usos del lenguaje de cada formato de juego según la siguiente clasificación funcional:

1. Reguladora, incluye habilidades comunicativas relacionadas con el control de la propia conducta y la de los demás.

2. Imaginativa, incluye habilidades comunicativas que crean un nuevo contexto modificándolo.

3. Obtener información, relacionada con habilidades comunicativas utilizadas como medio para obtener información en un sentido amplio (información del otro, del contexto, de lo que se está diciendo...).

4. Expresión, incluye habilidades comunicativas que aceptan o rechazan la intención del emisor.

5. Respuesta, surge durante el discurso a partir de las preguntas solicitadas por el otro interlocutor.

6. Informativa, incluye habilidades comunicativas que informan sobre un objeto, acción, suceso... y/o amplían la información anterior.

En segundo lugar, partiendo del concepto de comunicación como intercambio de significados, se cuantificaron el número de intervenciones de cada intercambio para determinar si existen diferencias cuantitativas en cuanto a la longitud del intercambio en función del tipo de juego. Se consideró por finalizado un intercambio cuando la pausa entre producciones era superior a 7 segundos.

Todos los registros se realizaron en vídeo, en la escuela infantil donde asistían los niños, con una duración de 15 minutos cada situación de juego.

\section{Formatos de juego}

Las observaciones se registraron a partir de dos formatos de juego. En la primera, los niños jugaban en una misma mesa pero con diferente material; se seleccionaron dos situaciones: una en la que el material era una caja de plastilina y pinturas de cera y folios; en la otra, el material era una caja de piezas de construcción y diversos puzzles. Cada uno de los miembros jugaba con uno de los dos materiales y podían cambiarse el material cuando quisieran. En el otro formato, las parejas tenían que jugar con el mismo material. Se seleccionaron tres situaciones de las que debían elegir dos. Una, en la que los niños jugaban con una cocinita completa, una cesta, dos muñecas, un cochecito y telas de colores; otra, en la que debían hacer una carretera con piezas de cartón, pequeñas, de diferentes tamaños y colores; y la otra en la que debían construir una casa para un elefante a partir de espumas y telas de diferentes colores y tamaños.

\section{RESULTADOS}

Como dato significativo a tener en cuenta, aunque no formaba parte del objetivo inicial, fué la diferencia cuantitativa de intervenciones respecto al tipo de juego. A los 3-3 1/2 años, el número total de intervenciones verbales fueron 87-94 en el formato de juego con diferente material, frente a 161-151 en el formato de juego construir una carretera y cocinita respectivamente. A los 4-4 1/2 años, los resultados fueron 76-69 intervenciones verbales en el formato de juego con diferente material frente a 153-163 en el juego de cocinita y construcción de una casa respectivamente. 


\section{Diferentes tipos de diálogos}

Haciendo una aproximación a la tipología característica de diálogos creados según el formato de juego registrados, y tomando como referente las características más significativas, los resultados muestran que, a los 3- 3 años y medio y en el formato interactivo con diferente material, los niños empiezan los intercambios con habilidades comunicativas que regulan la conducta del otro (llamar la atención del otro para enseñarle lo que hace o acaba de hacer), y se construyen a partir de intervenciones verbales que solicitan información, mayoritariamente en forma de pregunta y producciones que expresan aceptación o rechazo respecto a lo que se ha dicho anteriormente. Examinemos dos secuencias extraídas de dos registros, Xisco está pintando e Isaac jugando con plastilina

I. Mira un caragol/ ( «mira un caracol», mostrando a Xisco).

$\mathrm{X}$. No són així es caragols/ ( «no son así los caracoles», mirando a Isaac).

I. Que sil (mirando a Xisco).

X. Pintaré verd/verd/verd// (cogiendo una pintura).

Isaac juega con puzzles y Xisco con piezas de construcción

I. Nooo! sa paret no, sa paret no Xiscol («no! la pared no, la pared no Xisco», mientras mira a $\mathrm{Xisco)}$.

X. Tenim que pintar aquest/ ( «tenemos que pintar este», mientras coloca un muñeco).

I. Què?/ (mirando a Xisco).

$\mathrm{X}$. Tenim que pintar/ («tenemos que pintar», mientras coloca una pieza).

I. Com?/ ( ¿cómo?», mientras coloca una pieza del puzzle).

$\mathrm{X}$. Amb es pinzell/ («con el pincel», mientras encaja una pieza).

I. Quin pinzell?/ ("¿qué pincel?», coloca una pieza).

$\mathrm{X}$. Aquest que li don jo/ («este que yo le doy», mientras hace el gesto de dar al muñeco).

La primera secuencia forma parte del $85 \%$ de los intercambios verbales producidos. Dado el formato de juego, donde cada participante organiza su actividad de forma independiente sin necesitar la colaboración del otro, parece evidente que los propósitos del niño para usar el lenguaje serán fundamentalmente para mostrarle al otro lo que está haciendo. A nivel estructural, estos intercambios tienen una longitud de tres o cuatro movimientos (intervenciones), alternando habilidades comunicativas relacionadas con la función expresiva y de regulación de la conducta del otro y, con menor frecuencia de demandas de información.

La segunda secuencia forma parte del $15 \%$ de los intercambios producidos en este formato de juego. Son secuencias más largas construidas mayoritáriamente a partir de intenciones comunicativas de demanda de información verbal en forma de pregunta directa y respuestas. La secuencia Pregunta- Respuesta es característica en los intercambios más largos en este tipo de juego.

Veamos lo que ocurre en el formato de juego donde los participantes tienen que construir juntos una carretera

X. Jo posaré un quadrat/ ("yo pondré un cuadrado", coge y coloca).

I. Sí un quadrat/( «sí un cuadrado», mientras mira la carretera).

$\mathrm{X}$. Jo bo posaré aquílara n'Isaac té que posar un quadrat/cerca un quadrat/ ("yo pondré aquí/ahora Isaac tiene que poner un cuadrado/busca un cuadrado», coloca, señala a Isaac).

I. Ara tu bas de fer... un rotlo/ ( «ahora tú tienes que hacer un redondo», mirando a Xisco).

$X$. A ra toca a n'Isaacl («ahora toca a Isaac», señalando a Isaac).

Mayoritariamente ( $76 \%$ ), los inicios de las conversaciones están formados por un tipo de significado característico: usos comunicativos relacionados con la función de regulación, ya sea la propia acción o la del otro. Los intercambios comunicativos que aparecen con mayor frecuencia son los formados por cinco movimientos, y se van construyendo a partir de usos comunicativos que regulan mutuamente la acción. 

muñeca

Diferencias significativas aparecen en el formato de juego con cocinita y

I. I ara farem es dinar a sa fieta... i noltros li donarem/ («y ahora haremos la comida a la nena...y nosotros se lo daremos", coge un cuchillo y una cuchara).

X. I noltros li donarem amb sa cueral ("y nosotros se lo daremos con la cuchara», mirando a Isaac).

I. I sa forquilla què?/ ("¿y el tenedor qué?", mirando a Xisco).

$\mathrm{X}$. Amb sa forquilla noljo bo pos aquí damunt perquè no me fa faltal («con el tenedor no/yo lo pongo aquí encima porque no me hace falta", colocando el tenedor).

I. I a jo tampocl («y a mí tampoco», colocando el tenedor).

$\mathrm{X}$.Jo bo pos aqui damunt/ («yo lo pongo aquí encima», señalando con la mano).

Aparecen algunos intercambios más largos, aunque la mayoría sigue siendo como en el juego anterior,(5 intervenciones). Lo más destacado en estos tipos de diálogos es la presencia de una nueva función, la imaginativa, que va alternándose con usos comunicativos de obtención de información y de regulación de la propia acción.

Veamos lo que ocurre en los diferentes formatos de juego a la edad de 4- 4 años y medio. Atendiendo a los resultados obtenidos, el siguiente intercambio representa el modelo habitual de las conversaciones tipo que aparecen en el formato de juego donde cada participante juega con diferente material

Imma está jugando con puzzles y Laura con construcciones

I. Laura mira açò, Laural («Laura mira esto, Laura» mostrando una pieza de puzzle).

L. Eh?/ (mientras sigue jugando).

I. $U u u u u /$ (mientras arrastra la pieza por encima de la mesa).

La longitud media de los intercambios es de 2 y 3 intervenciones y, al igual que los intercambios comunicativos a los 3 años, los inicios están caracterizados por intenciones comunicativas que regulan la conducta del otro, y se estructuran a partir de habilidades comunicativas de obtención de información.

Por lo que respecta al formato de juego de cocinita, veamos una secuencia

I. Què vol?/ ("¿qué quiere?", mirando a Laura).

L. Em...posi...dos, dos palos de...de mantequilla/(«me ...ponga...dos, dos palos de...de mantequilla», mirando a Imma).

I. Vosté de mantequilla?/ («¿usted de mantequilla?», señalando a Laura).

L. $S_{i l}$ (mirando a Imma).

I. No en tenim no/ («no tenemos no", mirando a Laura).

En este formato aparecen intercambios formados por 5 y 6 intervenciones. Las habilidades comunicativas que aparecen con mayor.frecuencia son las imaginativas, creando y modificando el contexto de juego. Estas pueden ser afirmaciones/negaciones y/o demandas de información y respuestas. Lo mismo ocurre en el formato de juego de construcción de una casa, donde la diferencia respecto a aquel no es el tipo de habilidad comunicativa (sigue siendo la imaginativa alternándose con el habla que regula la propia acción), sino que, lo más característico es la extensión de los intercambios, todos ellos formados por 7 o más intervenciones, y éstas, a su vez, formadas por más de una expresión.

\section{CONCLUSIONES E IMPLICACIONES EDUCATIVAS}

A partir de los resultados obtenidos en este análisis de la comunicación verbal en la escuela, se confirma que los diferentes formatos de juego pueden considerarse como experiencias linguiísticas que dan lugar a diferencias en el uso del lenguaje en los niños pequeños, condicionando, a su vez, la longitud media del intercambio. 
Estas diferencias son significativas según sea el grado de participación conjunta y la edad.

Los intercambios comunicativos que se originan en las conversaciones infantiles a partir de diferentes juegos pueden convertirse en instrumento necesario para la práctica educativa. En primer lugar, si pensamos que la educación depende de la capacidad de los niños para utilizar el lenguaje de determinadas maneras, y, a la vez, el lenguaje es instrumento de comunicación que contribuye al pensamiento y al aprendizaje, la observación en clase de conversaciones en diferentes situaciones puede convertirse en un método válido para posibles valoraciones sobre la manera en que el niño uriliza el lenguaje en el trasnscurso de la conversación. La conversación espontánea puede convertirse en instrumento que ayude al niño a mejorar la expresión y a construir significados. El análisis posterior servirá para adoptar las correspondientes modificaciones que guiarán la práctica educativa. En segundo lugar, el formato de juego se convierte en instrumento capaz de modificar y provocar, por sí solo, diferentes maneras de utilizar el lenguaje. El repertorio de habilidades comunicativas del niño se adaptará a las exigencias y necesidades del juego. La organización y desarrollo del juego dependerá, evidentemente, de la distribución espacial y de los diferentes materiales (juguetes o no) que se dispongan en la clase, y también, cómo no, del número de niños implicados así como de la presencia del educador. La organización espacial del aula, así como la disponibilidad de materiales bien elegidos se convierten en elementos de reflexión en la práctica educativa para las actividades de enseñanza-aprendizaje de la comunicación verbal de los más pequeños. Los materiales invitan a jugar y a crear experiencias conjuntas, y, por supuesto, a conversar con ellos.

\section{Referencias}

Austin, J. L. (1962). How to do things with words. Cambridge Mass: Harvard University Press. Traducido: Cómo hacer cosas con palabras ( 1982). Barcelona: Paidós.

BRUNER, J. (1981). Acción, Pensamiento y Lenguaje. Madrid: Alianza Universidad.

GARVEY, G. (1984). El habla infantil. Morata. Madrid,1987.

HaLudDAY, M. A. K. (1975). Learning how to mean. Explorations in the Development of Language. Londres: Edward Arnold. Traducido: Exploraciones sobre las funciones del lenguaje (1982). Barcelona: Médica y Técnica.

PIAGET, J. (1975). Lenguaje y pensamiento en el niño. Buenos Aires: Guadalupe.

Rí DEL, M. J. (1993). Psicopedagogia del lenguaje oral: un enfoque comunicativo. ICE, Barcelona: Horsori.

Siguan, M.; Colomina, R., y ViLs, I. (1986). Metodologia per a l'estudi del llenguatge infantil. Barcelona: Eumo.

TOUGH, J. (1987). El lenguaje oral en la escuela. Madrid: Aprendizaje Visor.

TOUGH, J. (1989). Lenguaje, conversación y educación. Madrid: Aprendizaje Visor.

VYGOTSKI, L. S. (1979). El desarrollo de los procesos psíquicos superiores. Barcelona: Crítica. 


\section{Comunicación y juego. Usos del lenguaje infantil en diferentes situaciones de juego Pilar Nicolau CL\&E, 1995, 28, pp. 29-36}

Resumen: El objetivo fundamental planteado en esta investigación es analizar los diferentes usos del lenguaje en la comunicación verbal infantil en diferentes situaciones de juego. A partir de registros de situaciones interactivas de dos parejas de niños con edades comprendidas entre los 3- 3 años y medio y 4- 4 años y medio, se analizan las producciones verbales a partir de una clasificación funcional de 6 categorías. Los resultados muestran cuáles son las funciones más habituales que articulan el discurso del habla infantil a partir de diferentes materiales (juegos) de clase. En el último apartado, se derivan algunas implicaciones que pueden servir de reflexión para la práctịca educativa de la comunicación verbal infantil.

Datos sobre la autora: Profesora de Psicología Evolutiva y Didáctica de la Educación Infantil del Módulo Profesional «Educador Infantil», en el Instituto de Educación Secundaria Pascual Calbó en Mahón. Intereses actuales de estudio: psicopedagogía del lenguaje infantil y procesos de construcción del conocimiento en entornos educativos.

Dirección: Apartado de Correos 695.07760 Ciudadella (Menorca). Tel. 482210.

C PERMISOS PARA CITAR O REPRODUCIR EN OTRAS FUENTES: Se pueden citar libremente hasta 500 palabras. Para reproducir una porción de texto mayor, figuras o ilustraciones, se deberá pedir permiso por escrito a la revista, especificando el uso al que se destina el texto. En todos los casos, se deberá citar el copyright de $C L \& E$. En el caso de artículos o textos que hayan sido a su vez reproducidos en $C L \& E$ los interesados deberán dirigirse tanto a los detentadores del copyright original como a $C L \& E$, en el caso de que se quiera hacer uso de la traducción. FOTOCOPIAS: Para todo lo relacionado con el uso mediante fotocopia del material de esta revista, deberán dirigirse a: CEDRO, C/ José Marañón, 10, 3. Izda. Tel. 5941575 . Fax 4453567 